\title{
Socio-Cultural Factors Affecting the Treatment of Breast Cancer Among Pakistani Women and Potential Strategies to Prevent Breast Cancer: A Narrative Review
}

\author{
Gulshan Bano Ali ${ }^{1}$, Savera Aziz Ali ${ }^{2}$, Nadir Suhail ${ }^{3}$ and Sumera Aziz Ali ${ }^{4 *}$ \\ ${ }^{1}$ Centre for Epidemiology and Biostatistics the Melbourne School of Population and Global Health, Carlton, Australia \\ ${ }^{2}$ Department of Paediatric intensive care unit, Aga Khan University Hospital, Karachi, Pakistan \\ ${ }^{3}$ Department of Internal Medicine, Aga Khan University Hospital, Karachi, Pakistan \\ ${ }^{4}$ Department of Community Health Sciences, Aga Khan University, Karachi, Pakistan
}

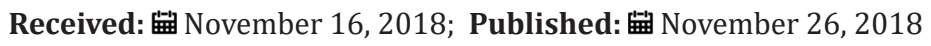

*Corresponding author: Sumera Aziz Ali, Assistant Professor, Department of Community Health Sciences, Pakistan

\section{Background}

The world health organization (WHO), since 1948, has defined health as "a state of complete physical, mental, and social wellbeing, not merely the absence of disease or infirmity". This was later expanded to include intellectual, environmental, and spiritual health. This is a very remarkable statement, considering that it was originally created in 1948(WHO) [1]. Personal responsibility is the driving force reflected in the above definition of health given by WHO, and the purpose defines destiny to achieve good health. If a state of ongoing health is our choice and purpose, then it is up to us to strive for this destiny ourselves. Life is a gift of God; the degree of health that we hold be a marker for how well we've taken care of this wonderful gift. Pakistan is ethnically, linguistically, religiously, and culturally a diverse society [2]. Our culture is a mixed culture. Pakistan is a Muslim country, although most people in Pakistan are Muslims, there is a strong influence of different cultures and their social activities on our culture, for example, Hindu culture influences are visible on our ceremonies and festivals. Despite significant progress has been made in recent years, the development indicators for Pakistan are still low. In addition, poverty is the major problem in our country, every person could not earn $1 \$$ daily according to WHO.

The country is largely comprised of scattered rural communities. The total expenditure on health from the gross domestic products is less than 2 percent and health services are mainly provided by the private sectors in Pakistan. The health indicators are very poor and current health status of the nation is characterized by a high population growth rate, high incidence of low birth weight babies and high maternal and neonatal mortality. In addition to these problems, both communicable and non-communicable diseases are increasing day by day. Communicable, infectious and parasitic diseases remain a severe burden on the country in the form of malaria and tuberculosis which continue to remain potential threats for the Pakistani Population [3]. Some of the health problems faced by the health sector are:

i. Inadequate primary health care

ii. A high rate of population growth

iii. Prevalence of communicable and non-communicable diseases

iv. Managerial deficiencies

v. The inadequacy of funding and trained manpower

vi. Centralized administrative and financial powers

vii. Lack of mass awareness of health.

\section{Introduction of Breast Cancer}

Breast cancer is a type of cancer which originates from breast tissue. The common site for the origination of breast cancer is the lining of milk duct or lobules. Breast cancer is the second leading cause of death in women both in developed and developing world. To detect the breast cancer in the modern time is completely different than it was in the past, because of medical advancements, many modifications are the consequences of what is called the 'Breast Cancer Advocacy Movement'. This movement came in two forms i.e. the first wave in the 1970s, represented breast cancer as "Public" and offered the asserting concept that "it's alright to have this cancer: you don't have to conceal it". The concept of the second wave was started in the 1990s, it stated that "it is not alright to have this cancer: we have to end it from occurrence". Previously diagnosis and treatment were not adequate that's why the objective must be the avoidance of this disease [4]. Now a day, proper techniques of diagnosis, as well as improved treatment of breast cancer, are available. 
The incidence of breast cancer is increasing in the developing world due to increasing life expectancy, increasing urbanization and adoption of the western lifestyle. Although some reduction has been achieved through prevention, these changes still cannot eliminate breast cancer that develops in low and middle-income countries. Worldwide around $16 \%$ of all female cancers are attributed to breast cancer. In 2004 an estimated 519000 women died because of breast cancer. $69 \%$ of all breast cancer deaths occurred in developing countries because of poor treatment facilities as well as delayed diagnosis. The incidence rate of this disease is 99.4 per 100,000 in North America. Eastern Europe, South America, South Africa, and Western Asia have moderate incidence rate, but still an increasing rate. The survival rate of breast cancer is varying greatly worldwide, ranging from $80 \%$ in North America, Sweden, and Japan to around $60 \%$ in middle-income countries and below $40 \%$ in lowincome counties. The low survival rate in less developed countries is because of late diagnosis as well as lack of adequate diagnosis and treatment facilities [5]. Nearly 1.4 million cases of cancer were diagnosed across the world in 2008 compared to about 500,000 cases in 1975 . This shows about $11 \%$ of all new cancer cases and $23 \%$ of all female cancers. It is predicted that the number of cases will rise to 2.1 million by 2030 [6].

In Pakistan, breast cancer cases are increasing all over the country. Although nationally representative data for breast cancer is not available, data from some provincial registries indicate that it has been on the rise. Approximately one in every nine Pakistani women is likely to suffer from breast cancer. This is the highest rate of incidence all over Asia. Which is 50 per 100,000 women while in India (with similar Socio-cultural background) the incidence rate is 19 per 100,000 [7]. At the Shaukat Khanum Memorial Cancer Hospital and Research Center in Lahore, Pakistan, cancer of breast has been registered as the commonest malignancy amongst women during a fifteen-year period extended from December 1994 till December 2009; here it accounts for nearly 46\% (8915) of the total malignancies in females diagnosed or treated at the hospital [8]. Evidence from the Karachi cancer registry indicates that the age-standardized annual rate of breast cancer is 69.1 per 100,000 . This rate is almost equal to European and North American rates [9]. According to the International Agency for Research on Cancer (WHO) GLOBOCAN 2008, the number of cases of breast cancer in Pakistan is 19271 (crude rate, 22.5) and the number of deaths is 10376 (crude rate, 12.1) [6].

\section{Secondary Data regarding factors affecting Breast Cancer}

The health of the women was never considered an important issue because a woman has a little respect in the community. In the name of culture, tradition, and religion they are never given equal status in the society. A system which is based on gender inequality will not adopt policies for wellbeing of women who are poor, powerless and weak as a class. Multiple studies have been done on breast cancer globally as well as in third world countries. These studies show similar indicators like mortality, morbidity rates, and factors which affects the prevention and treatment of breast cancer. For example, a study conducted in Belgium shows that socio-economic status and education level are linked with late diagnosis and poor treatment of breast cancer in the female. Socio-economically disadvantaged populations prove to have lesser access to proper health care and have more invasive carcinomas and higher mortality rates. The Belgium government study compared the results of the socio-economic status of patients in several hospitals in the country and made up a classification. The more obvious results were, the lower level of education, resulting in lower income, more patients were belonging to the special class of widows, orphans and most of the patients had no insurance at all [10]. These all factors act as barriers for treatment of this disease.

In 2010, an estimated 207,090 new cases of invasive breast cancer are expected to be diagnosed in women in the US, along with 54,010 new cases of non-invasive breast cancer [11]. The mortality rate of breast cancer increases because of factors affecting the treatment practices. A study conducted in the USA clearly shows that the education level of women is directly related to the treatment of breast cancer those who are highly educated took treatment like chemotherapy as compared to women with low education [12]. It also suggested that both low income and language barrier also affect the treatment of breast cancer. Another similar study was done on African-American women, to identify the effect of race and socioeconomic status on treatment and survival of breast cancer. This study also shows that females belonging to low socio-economic status have less accessibility to treatment [13].

\section{The Rationale for doing this Narrative Review}

As the above data of breast cancer depicts that the magnitude and severity of the disease are increasing very rapidly. In addition, this disease imposes a heavy burden on public health. It becomes a threatening disease to both developing as well as developed countries. It is the emerging health issue at the global level. Millions of females are victims of this disease. However overall mortality because of the breast cancer has been decreased despite that many females die every year. Moreover, not only the patient suffers but also the whole family is under pressure because of the economic burden and psychological issues faced due to this disease. Being a female, she has not only to run the family but also must take care of her children. Therefore, most of the time children suffer more than anyone else in the family as nobody can take care of them just like a mother.

It is well known that social class disparities result in inequalities in effectiveness of health care treatment and health care facilities even in developed countries. Socioeconomic factors such as lower income, level of education, cultural beliefs and screening practices appear to affect the treatment as well as survival [14]. Prognosis and survival rate are much better in developed countries due to early diagnosis and treatment. But in countries like Pakistan with limited resources and poor practices, the disease is usually detected at a later stage. At later stages treatment is not usually effective to cure the disease, hence decreases the survival period. In Pakistan, the incidence rate of breast cancer is much higher as 
compared to other Asian countries. Despite having good resources like mammography and other screening procedures, the mortality of breast cancer is still high in the country. Most of our population belongs to low socioeconomic status, therefore, they cannot afford such expensive procedures. In addition, health education is very poor among females living in rural areas; they do not prefer to go for screening and other treatment even in the worst condition. Female in our society is considered as a less autonomous and poor creature of God. They are subordinated and are not allowed to get a good education mainly in rural areas. They are underestimated by the family; because of unawareness they are unable to share their problems with spouses and other family member and cannot take any decision about the treatment. Due to these issues, in our society, diseases related to female personal organs increases very rapidly e.g. breast cancer, cervical cancer, and ovarian cancer.

Very few studies have been conducted in Pakistan on factors affecting treatment of breast cancer. One such study concluded showed that there are so many factors which upset the patients psychologically and prevent them to take treatment. One of such factors is the financial burden of treatment, which is a common theme related to the financial implications of treatment in Pakistan. Many families using all their savings to pay out of pocket for treatment, this was often related to loss of breadwinners and income in the family. For younger, unmarried women, the cost of treating breast cancer meant that families could not offer financial assistance for dowries [9].

Another study done in Pakistan shows that our culture is also responsible for severely unhealthy conditions. As women feel that having breast cancer is associated with issues of shyness, personal modesty, and embarrassment, therefore, breast always had to be concealed. These factors increase resistance to openly discuss issues related to breast between women, mothers, daughters, and spouses or other family members. Thus, it shows that breast cancer is a socially unacceptable disease. Because women in our society are told from an early young age that this organ is to kept secret, so they don't talk about it very openly with their family members unless they only talk about with their friends, who are not very much aware of the disease. Hence everyone tries to keep it hidden. That's why they are diagnosed at the fourth stage when there is no option except surgery [15]. In case of surgery, women do not prefer a male doctor due to shyness.

\section{Socio-Cultural factors affecting the treatment of Breast Cancer}

Socio-cultural determinants play a very important role in the diagnosis, treatment, and survival of breast cancer. Social determinants have been called the fundamental cause of health and disease. As we can see the incidence of breast cancer is increasing in our society. There are several socio-cultural determinants which can affect the treatment of breast cancer in one or other way. Some of those factors are lack of education and unawareness, socioeconomic status; gender bias, ignorance, fear regarding cancer survival, misinformation from family and friends, the decision regarding the treatment. Some of these factors are mentioned here.

\section{a) Lack of Education and Unawareness:}

The first and foremost method to prevent oneself from the diseases is the awareness about the disease. Education is the key to face the challenges of life across the globe. Lack of education and unawareness about breast cancer, the importance of early detection and other factors affect the treatment of breast cancer. In Pakistan, illiteracy rate is high in women, which is responsible for their poor health. In rural areas female ignore their health because they are responsible for taking care of family and home. They must take care only of house chores and children that's why they don't need education and the only male can go for getting an education because he must support the family financially. These misconceptions leave females behind in education than males. Thus, they are unable to get proper education and are unaware of health problems and its treatment. Once the females are uneducated, they also psychologically think that they are illiterate, their ideas will not be given any value in the society. Naturally, when female is not aware of any tumor, how can she realize that she is suffering from some problem. Because it is not anyone else only the woman herself is the first and right person to diagnose the disease, especially diseases regarding the breast and any other personal organ. Due to this factor of knowledge their disease is not diagnosed properly at the right time. In the end, not only the woman but also the whole family has to face such problems. This is the reason how their treatment regarding breast cancer is affected. Also because of lack of education women feel shy to share personal health problems and most of the time the women underestimate themselves in the family and hesitate to share their problems. A vicious circle of the problems starts without having an end.

\section{b) Socio-economic Status:}

"If you don't have money for treatment, you don't want to know if you are sick". As this saying shows, low socioeconomic status seems to be the primary social factor affecting treatment of breast cancer. Poverty is linked to decreasing the rate of breast cancer screening, late-stage diagnosis, inadequate treatment, and higher mortality. Patients find it too hard to go to the doctor for treatment because of bills. It is not only the direct cost of the disease but so many indirect expenses are there including time of patients' and their family members. In Pakistan, more than half of the population belongs to low socioeconomic status. They cannot even afford three times food for their daily intake and it's very difficult for them to go for health care. Especially women are more concerned about the financial problems faced by them. In our society, where the women are not earning, therefore in the starting of the month, they are given a scarce amount of the money and are asked to not demand anything extra before the completion of the month. They are more conscious about their children and other family members while they do not prefer themselves and hide their health problems.

In addition, the women do not bother to share their issues with the family members unless they do not experience severe pain. In poor families even if they complain about their pain, family members do not show any concern about treatment because of money. As we know overall health is considered as shopping in our country. This 
means you pay for health and you buy it. As everybody wants to buy the quality care but unfortunately not everybody has access to that. Therefore, poor people suffer a lot. Many factors are associated with poverty and poverty itself is a big barrier in the treatment of breast cancer. Sometimes in rural areas, people start selling their cattle, all the household things and personal belongings just because they want their loving ones to be taken care of properly. Like the other rich men of the society, they also have emotions and feelings about the females and they want to take every possible step to save the lives of their women. But despite putting a lot of efforts, they give up at the end because of the financial issues which drive them to catastrophic expenditures and impoverishment.

\section{c) Gender bias:}

Once the factors regarding poverty and education are resolved, the issue arises of getting treatment from whom. There is no doubt that historically, women in our society have suffered several forms of discrimination. Female patients of breast cancer think 1000 time before going for treatment because most breast cancer surgeons are male in Pakistan. There are so many reasons for this issue, some female does not prefer to go to a male doctor for treatment because of their own values. Sometime husband and family members are not willing for the male surgeon, due to which females have to suffer more. Because of the low level of education and religious values in our country, people avoid male doctors thus creating a big hurdle in the treatment of breast cancer.

In addition to this, in our society, females are subordinated, who has less power, having no right to taking any decision even for their lives. They are always dependent on their husbands or parents to take any step of their life, they must follow their dominant family members whatever they decide about their lives. Especially in rural area females are not allowed to go out of home alone for any purpose, which makes their life very difficult. They don't have the power to take a decision about their health because of the pressure and fear from family. If they suffer from breast cancer their male partner will not allow them for surgery. In addition, probably males do not want to see their female without a breast. Hence, they ignore her health for the sack of their desires.

\section{d) Fear regarding Cancer Survival:}

Breast cancer involves multidisciplinary collaboration. The patients should be aware of both the beneficial and adverse effects of the different treatment options to make a well-informed decision. For treating any patient, it is very important to have patients preference. Breast surgery is the best way to treat local disease, but the major issue arises here is the fear of patients regarding survival. As we know females are weak that's why they feared about such treatment. It is well known that cancer is a lethal disease and it cannot be easily cured, either chemotherapy or surgery is required for cancer cases. Before any treatment, doctors make aware the patient about all types of side effect and benefits to take the consent from the patient. After knowing the side effects of any treatment for breast cancer, most of the females refuse to take any treatment. This further adverse the condition, and finally the patient dies just because of fear of survival. In our community diagnosis of breast cancer often means a death sentence. This is true for people with few financial resources. Also, the individuals may fear the discovery of these cancers and worries about seeming defective or less feminine to a partner and disfigurement. These emotions, combined with the cost of health care, limited accessibility, and the daily need to care for a busy life, may lead many patients to postpone treatment.

\section{e) Wrong information from family and friends:}

In our society, sometimes it happens that some members from the family or friends provide wrong information regarding treatment of breast cancer. This wrong information creates a lot of confusion for the patient about the treatment. As we all know the side effects of chemotherapy are adverse, it causes patients hair fall and patient may become very weak physically. In some communities where the education level is very low, people expect that the treatment is not good, doctors are giving the wrong treatment which is responsible for such adverse conditions. When the woman with breast cancer comes to know about such misconception, she refuses for such treatment even if the disease is in the late stage.

\section{f) The decision regarding treatment:}

As we know that in our society most of the time decision regarding important matters are taken by the elder members of the family. In our culture, either mother in law or husbands has the right to decide about the treatment of any disease of the female. The suffering woman is silent and cannot even participate in the decision taken about her own life. She is not in the position to take an early step regarding the treatment of her disease. From the treatment point of view breast cancer is a very sensitive issue. If the decision about the treatment is not taken early, then woman suffers a lot. As we know that breast cancer treatment has multiple steps. It is not only the surgery where the story stops; sometimes it becomes a lifelong issue for the woman. Female needs social, moral and psychological support from the family. Therefore, family members have to think for so many times before taking any right decision. But unfortunately, by the time they take a decision about the female, it becomes very late and disease is spread to the whole body.

\section{Strategies and Interventions to Prevent and Cure Breast Cancer}

Breast cancer is the most common cancer in women in the East Mediterranean Region and the leading cause of mortality worldwide. There is geographic variation, with the standardized age-incidence rate being lower in developing than industrialized countries [16]. Breast cancer detection and prevention is a systemic and continuous management process that includes planning, developing and evaluating breast cancer detection programmes, including policy formulation and the identification of priorities. Every country should develop proper plans for screening and early detection, including education of the general population, training for technical and medical staff develops programs and processes for accurate diagnosis and facilities for timely and effective treatment. 
There so many practices which can help in the prevention of breast cancer e.g. surveillance, protection, continuing education, and prevention, early detection and care. Some of these strategies and interventions are given below:

\section{Surveillance}

Surveillance is the key for identifying health problems and helps in developing appropriate interventions. Before going for intervention there should be surveillance. It helps in estimating the burden of the disease on society, identifies the risk factor which increases the incidence of the disease and it builds the basis for appropriate clinical interventions.

\section{Protection}

It comprises many activities and processes associated with protecting individuals from breast cancer and its reoccurrence such as such as education, health promotion, prevention, and early detection e.g. screening. Protection is the first step in the prevention of any disease.

\section{i. Continuing education}

The first step in any intervention is the development of the education programs for the general population. Government officials and policymakers can place the health education programs on the country's national agenda. Public education programs should focus on prevention, a better understanding of the illness and the benefits of early detection. These programs can provide awareness to the recipients and their families about health problems and their solution. With the help of such activities, we can easily understand the needs of patients and can easily cope with the situation. It makes aware people about the risk factors of breast cancer, which can easily be controlled.

\section{ii. Prevention}

Although breast cancer cannot be prevented, the risk factors of breast cancer can be reduced through specific preventive activities. These include changes in lifestyle, diet modification, physical activity and obesity, and interventions for women at high risk of developing breast cancer using drugs like tamoxifen and other antiestrogen compounds.

\section{iii. Early detection}

The most important and beneficial area of protection activities in the early detection of breast cancer. Screening is the most important tool of breast cancer, which diagnosis disease in its early stage. It decreases the mortality and morbidity of the illness. There are several practices for the screening of breast cancer including breast self- examination, clinical breast examination and mammography. The effectiveness of these all procedures depends on education, a skill of health workers and facilities and the equipment used, skills of technician and radiologist reading the mammogram respectively.

\section{iv. Care / disease intervention}

Once the disease is diagnosed there should be proper treatment available. Treatment is more effective when the disease is diagnosed in early stages. If the disease is diagnosed at a late stage, improving treatment and care provided to the women with breast cancer is obviously an integral factor in decreasing overall mortality from the disease. Treatment of breast cancer should be expanded beyond surgery to include interventions such as drug therapy and radiation procedures [17].

\section{Intervention Option for Factors Affecting Treatment of Breast Cancer}

Over thelastseveralyears, numerous preventive and therapeutic interventions have been introduced with the aim of helping women suffered from breast cancer. As we can understand from the above discussion that the intervention options for overall breast cancer prevention are somehow different in different settings, but the aim is similar across all settings.

\section{i. Individual-level approach}

Females have the same rights for education as males. Illiteracy is the major factor which is responsible for all type of unhealthy conditions. An educated person knows very well about their needs and rights. They can only be able to take a decision about their treatment options when they will be provided with proper education. Once females get the education, they will become aware of anything related to their wellbeing. There should be campaigns which provide proper knowledge about the breast cancer to every individual female not only those suffering from breast cancer but also other healthy women in the community. Extensive awareness programs can help in reducing these factors affecting the treatment of breast cancer.

\section{ii. Family level approach:}

Chronically ill patients, especially with life-threatening diseases like breast cancer, went through a series of stressful life events. Evidence suggested that the availability of social support during times of stress may increases survival from cancer. Families are responsible for the better treatment of Patients having breast. Female cannot take a step for treatment alone; she needs support from the family members. We develop programs for the awareness of people living in rural areas, most of them are illiterate and they do not know about the diseases as well as the treatments. We should create awareness among the families to support the patient psychologically, morally and financially to take good treatment for such a lethal disease as possible as in the early stages.

\section{iii. Organization and population level approach:}

Different organizations and the communities both should put efforts for prevention of socio-cultural factor which affect the treatment of breast cancer in our country. There is a big issue that female patients of breast cancer do not prefer to take treatment from a male surgeon. There should be good scholarships to female students for specialization. In our country there are very few female surgeons are available for surgery, it makes the situation very critical. Treatment of breast cancer is very expensive, the government should introduce new, low costand effective treatments options for the patients belong to low socio-economic status. 


\section{Conclusion}

In conclusion, females in our community are taking care of maternal responsibilities for sake of their health, which seems to be an innate characteristic of Pakistani women. But our culture is subordinated with women which creates differences in their need and place in society. Due to such differences, females are not given the chance to get her rights. They are unable to take care of their health and suffered from lethal disease like breast cancer. Our government should take this issue seriously and make some efforts to provide rights for the women. Now a day different nongovernmental organization are working for the rights of women and the Pakistani government should collaborate with them. In the case of breast cancer, there should be proper campaigns in rural settings that the female will become aware of their disease and will be able to take decision for good treatment.

\section{References}

1. http://www.who.int/about/definition/en/print.html (2010).

2. (2009) Pakistan: Plan 2010-2011 (MAAPK002) source: International Federation of Red Cross and Red Crescent Society (IFRC) cited from.

3. Executive summary of health system profile- Pakistan, Regional health system observatory- EMRO.

4. History, facts and statistics.

5. Breast cancer burden (WHO).

6. (2008) International agency for research on cancer (WHO), GLOBOCAN, incidence and mortality worldwide.
7. Naeem M, Khan N, Aman Z, Nasir A, Samad A, et al. (2008) Pattern of Breast Cancer: Experience At Lady Reading Hospital, Peshawar. Journal of Ayub Medical College Abbottabad 20(4): 22-25.

8. Nadir Quershi (2011) Collective cancer registry report from dec. 1994 up till dec. 2009, Source: Cancer Registry \& Clinical Data Management, Shaukat Khanum Memorial Cancer Hospital and Research Center.

9. Banning M, Hafeez H, Faisal S, Hassan M, Zafar A (2009) The Impact of Culture and Sociological and Psychological Issues on Muslim Patients with Breast Cancer in Pakistan. cancer nursing 30(4): 317-324.

10. Cuyper LD, Robrecht H, Sterckx F (2003) The Effect of Social Factors on Diagnosis and Treatment of Breast Cancer. Acta Chir Belg Journal 103: 585-588.

11. (2018) Breast cancer statistic, BREASTCANCER.ORG.

12. Morimoto L, Jenna C, Fionna M, Cynthia O (2010) Factors Affecting Receipt of Chemotherapy in Women with Breast Cancer. International Journal of Women's Health 2: 107-122.

13. Cathy J, Given CW, Roberts C (2002) "Race, Socioeconomic Status, and Breast Cancer Treatment and Survival." Journal of the National Cancer Institute 94(7): 490-496.

14. Aziz Z, Sana S, Akram M, Saeed A (2004) "Socioeconomic Status and Breast Cancer Survival in Pakistani Women." Journal of Pakistan Medical Association 54(9): 448-453.

15. Banning M, Haafeez H (2010) A Two-Center Study of Muslim Women's Views of Breast Cancer and Breast Health Practices in Pakistan and the UK. Journal of Cancer Education 25(3): 349-353.

16. EMRO Technical Publication Series 31, guideline for management of breast cancer.

17. EMRO Technical Publication Series 30, guidelines for the early detection and screening of breast cancer. Submission Link: Submit Article

DOI: $10.32474 /$ OAJRSD.2018.02.000126
This work is licensed under Creative Commons Attribution 4.0 License 